Gut, 1972, 13, 54-57

\title{
Plasminogen activator in bile during extracorporeal perfusion of pig liver
}

\author{
A. JEDRYCHOWSKI, ${ }^{1}$ S. P. PARBHOO, AND P. HILLENBRAND ${ }^{2}$ \\ From the Departments of Medicine and Surgery, Royal Free Hospital, London
}

SUMMARY Plasminogen activator activity was studied in bile and perfusate during extracorporeal pig liver perfusion. Plasminogen activator activity in bile was up to 58 times greater than in perfusate. A high level of plasminogen activator activity in bile was also found after the addition of urokinase to the perfusate. These results suggest that bile is the main route of active excretion of plasminogen activator and urokinase from the liver in experimental liver perfusion.

Clinical and experimental data suggest that the liver is an important clearing site for plasma plasminogen activator (Kwaan, McFadzean, and Cook, 1957; Fletcher, Biederman, Moore, Alkjaersig, and Sherry, 1964; Kulapongs and Bachmann, 1968; Januszko, 1969). The mechanism of this clearance is unknown, although there is some evidence that urokinase, when added to the circulation in rat liver perfusion, is cleared in the bile (Kulapongs and Bachmann, 1968). In this paper we present the results of investigations of plasminogen activator in perfusate and bile produced during extracorporeal perfusion with pig liver.

\section{Techniques and Methods}

The liver was perfused using the same technique as in clinical liver perfusion (Parbhoo, Kennedy, James, Chalstrey, Ajdukiewicz, Brock, Xanalatos, Sayer, and Sherlock, 1971), except that no connexion was made to a patient. The perfusion apparatus was designed to provide optimum conditions for the function of the isolated liver for at least eight hours. Approximately 2.5 litres of human blood or bloodless perfusate were used in each experiment.

Plasminogen activator activity in plasma and bile was studied during four perfusions. The first two perfusions were performed under standard conditions. In the third perfusion, urokinase (Leo Lab. 15,000 Ploug units) was added to the perfusate one hour after starting perfusion. A bloodless perfusate

\footnotetext{
${ }^{1}$ British Council scholar

${ }^{2}$ Stanley Thomas Johnson fellow

Received for publication 1 November 1971.
}

(balanced, buffered, oncotically normal electrolyte and dextran solution) was used instead of blood in the fourth perfusion to assess the effect of hepatocellular damage.

Fibrinolytic activity was measured using the euglobulin test and the fibrin plate method. The euglobulin test in plasma was carried out according to the method of Januszko and Dubinska (1965), the plasminogen activator activity being expressed as units per $\mathrm{ml}$ derived from the formula $1300 / \mathrm{t}$ where $t$ is the euglobulin lysis time in minutes. The experimental procedure for the estimation of plasminogen activator in bile was as follows: double dilutions of $0.5 \mathrm{ml}$ of bile were made in $0.5 \mathrm{ml}$ of normal saline, dilutions ranging from $1: 2$ to $1: 64$. One $\mathrm{ml}$ of standard human plasma was added to each test tube. The control tube contained $1 \mathrm{ml}$ of standard human plasma and $0.5 \mathrm{ml}$ of normal saline. Euglobulins were prepared separately from each sample (containing a mixture of bile and standard plasma) according to the above mentioned method. Plasminogen activator activity was calculated from the formula P.a a. $=\frac{1300}{t}$, where $t$ is the euglobulin lysis time in minutes. The plasminogen activator activity of each euglobulin sample was compared with that of the standard plasma and the mean value of plasminogen activator activity per $\mathrm{ml}$ of bile was calculated using sample dilutions 1:4-1:32. The procedure for determining plasminogen activator in the bloodless perfusate was that used for the estimations in bile.

In order to exclude the possibility that lysis was partly due to bile salts, unheated, calcium-containing fibrin plates $(0.2 \%$ fibrinogen) were prepared 
(Fleming and Norman, 1960). These plates are resistant to the direct lytic action of bile salts. We found no lysis when incubating pig bile obtained from the common duct on heated plates. There was also no lysis when heated bile $\left(85^{\circ} \mathrm{C}\right.$ for 30 minutes) was incubated on either heated or unheated fibrin plates. The effect of bile salts in the bile was tested in the euglobulin test by the fact that euglobulin from standard human plasma plus bile did not cause lysis on heated fibrin plates. These findings proved that lysis, which occurred only on unheated fibrin plates, was due to plasminogen activator activity. The area of lysis on unheated fibrin plates ranged from 150 to $250 \mathrm{~mm}^{2}$ using pig bile obtained from the common duct. These results corresponded to 17-40 units in the euglobulin test.

\section{Results}

There was close correlation between the results of the fibrin plate method and the euglobulin test. We found it more convenient to express the results using the euglobulin test and therefore only the results obtained by this method are presented here.

Plasminogen activator activity per $\mathrm{ml}$ of bile ranged from initial values of 7 to 70 units to peak values of 580 to 980 units) As the bile flow fluctuated throughout perfusion, the total activity in each collection period was calculated in order to give a better measure of overall activity of plasminogen activator. This was done by multiplying the activity of plasminogen activator in units per $\mathrm{ml}$ of bile by the volume of bile secreted in each 30 - or 60-minute collection period. Half-hourly and hourly values are shown in Figures 1 and 2.

In experiments 1 and 2 (Figs. 1a and $\mathrm{b}$ ) peak bile flow occurred between the second and fourth hours of perfusion. Total plasminogen activator activity in each collection period increased from initial values of 8 and 62 units to peak values of 2,540 and 6,090 units during the fourth and fifth hours of perfusion.

In experiment 3 (Fig. 1c), in which urokinase was used, the secretory pattern of plasminogen activator was similar to that in experiments 1 and 2 . However, the peak level, as well as the overall amount of plasminogen activator excreted into the bile throughout the perfusion was greater than that in experiments 1 and 2.

In experiment 4 (Fig. 2) very little bile was produced and only a small amount of plasminogen activator was excreted into the bile, with a peak output at 60 minutes.

In Fig. 3, activity of plasminogen activator in plasma (experiments 1-3) and in the bloodless perfusate (experiment 4) is shown. As can be seen on this graph, the peak of plasma activity of plasminogen activator occurs in the first $\mathbf{3 0}$ minutes of perfusion and falls rapidly in the next 30 minutes,

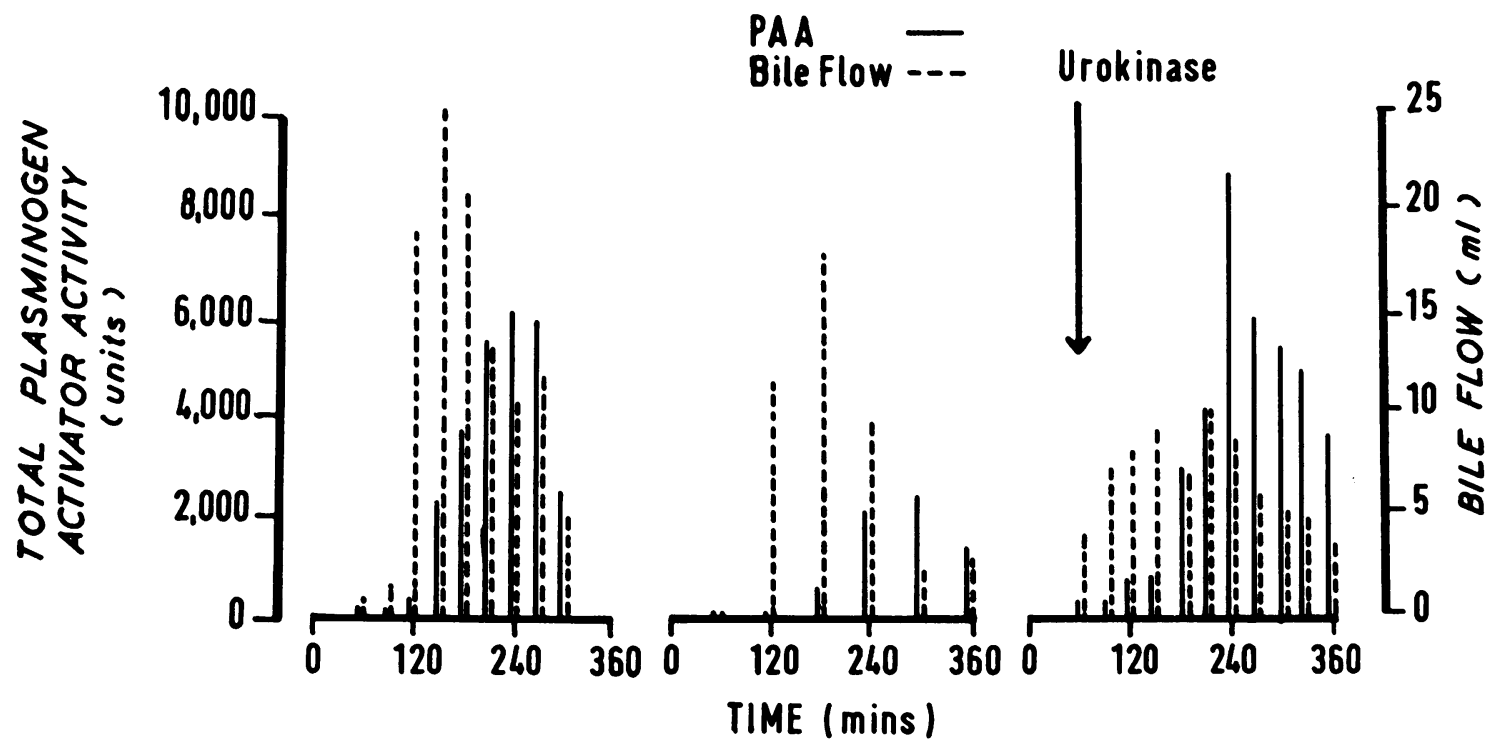

(a)

(b)

(c)

Fig. 1 Total plasminogen activator activity in bile and bile flow.

Fig. 1a-b Perfusion with blood (experiments 1-3)

Fig. 1c Urokinase (15,000 Ploug units) added at 60 minutes. 


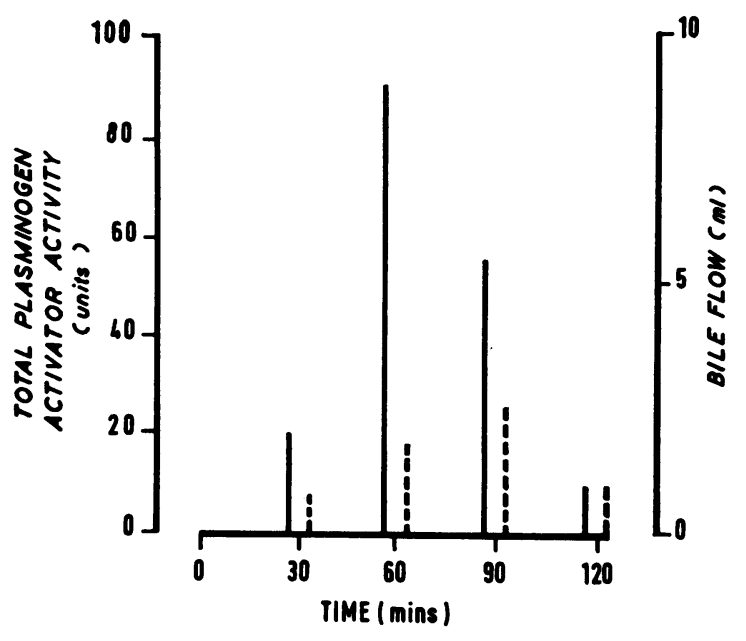

Fig. 2 Total plasminogen activator activity in bile and bile flow: perfusion with electrolyte and dextran solution (experiment 4).

returning gradually to pre-perfusion levels thereafter. There is an additional peak in experiment 3 due to the addition of urokinase. In experiment 4 there was also a peak in the first $\mathbf{3 0}$ minutes, but activity declined rapidly and was undetectable after one hour.

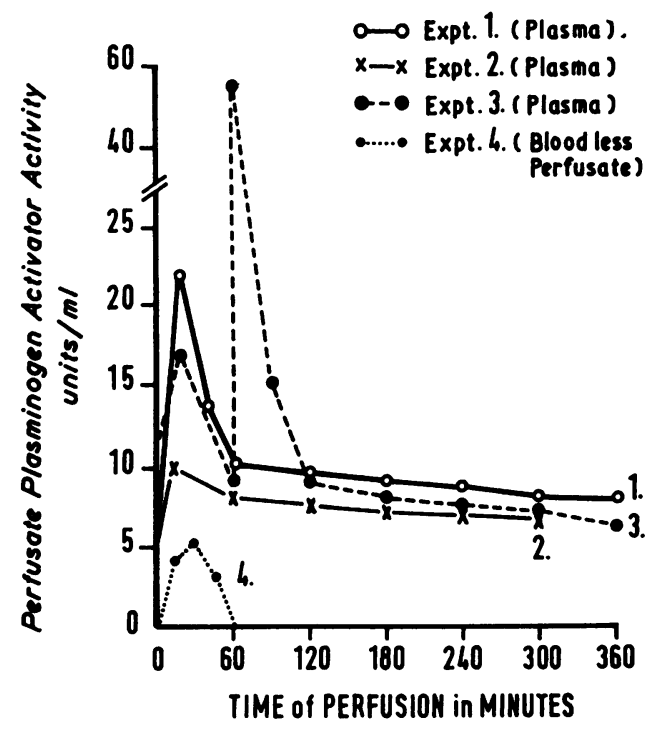

Fig. 3 Perfusate plasminogen activator activity (experiments 1-4): urokinase 15,000 Ploug units added at 60 min in experiment 3.

\section{Discussion}

In our previous perfusions we found that the increase in plasma plasminogen activator activity during the first hour of perfusion was due to the release of plasminogen activator from the liver (probably from the blood vessels). It is then rapidly cleared by the liver and it has been shown that its removal via the ascitic fluid and lymph is insignificant; the plasminogen activator activity in these fluids was lower than in plasma (unpublished data). However, in the present study, when the peak values of plasminogen activator activity are compared (Table), its activity in bile produced during perfusion was up to 58 times greater than the plasma activity. Furthermore, in experiment 1 , plasma plasminogen activator activity was higher than in experiment 2 , and plasminogen activator in bile was also correspondingly higher in the first experiment than in the second. These results suggest that bile is the main route of active excretion of plasminogen activator from the blood.

\begin{tabular}{llc}
\hline No. of Experiment & \multicolumn{2}{l}{ Plasminogen } \\
\cline { 2 - 3 } & Perfivator $(u / m l)$ \\
\hline 1 & 22 & Bile \\
2 & 10 & 977 \\
3 & $17^{1}$ & 580 \\
4 & $59^{2}$ & 980 \\
\hline
\end{tabular}

Table Peak values of plasminogen activator activity in perfusate and bile during extracorporeal pig liver perfusion

${ }^{1}$ Peak value before adding urokinase to perfusate ${ }^{2}$ Peak value after adding urokinase to perfusate

It is unlikely that the increase in plasminogen activator activity in the bile is due to hepatocellular damage during perfusion, as activity declines in the later stages of perfusion. This view is supported by the findings in experiment 4 , when, after a bloodless perfusion, very little plasminogen activator was excreted into the bile from the ischaemic liver.

The results of the experiment with urokinase (Fig. 1c) also indicate that there is active excretion of plasminogen activator in the bile. There was an increase, compared with experiments 1 and 2 , in both the peak activity of plasminogen activator in bile and in the overall amount of plasminogen activity. Fibrinolytic activity in bile after the addition of urokinase to the circulation was investigated by Kulapongs and Bachmann (1968) in an isolated rat liver perfusion system. These authors found a twoto three-fold increase in fibrinolytic activity in bile compared with the perfusate, whereas in our experiments there was a 16-fold increase. 
As can be seen in Figs. 1a-c, the peaks of bile flow and of plasminogen activator activity do not coincide. This strengthens the evidence for active excretion of plasminogen activator in the bile and suggests that its excretion is unlikely to be simply a washout phenomenon.

Plasminogen activator has been found in the bile of animals and man (Kulapongs and Bachmann, 1968; Fleming and Norman, 1960; Hata, 1967; Oshiba and Schoenfield, 1970). However, there has been little work investigating the significance of the biliary system in the hepatic clearance of plasma plasminogen activator. From the data in this study it may be concluded that plasma plasminogen activator and urokinase are preferentially excreted into the bile and that biliary excretion is therefore an important mechanism in the removal of plasminogen activator from the circulation. The presence of plasminogen activator activity in bile suggests that plasminogen activator may play a part in maintaining the patency of the bile ducts. Further studies are required in man to determine the extent and significance of excretion of plasminogen activator in bile.

We are grateful to Miss Lesley Halliwell for technical assistance. This work was supported by the Royal Free Hospital Endowment Fund, the Ingram Fund,
Davies Memorial Fund, and a Peter Samuel Trust grant to S.P.P. We are indebted to our colleagues at the Royal Veterinary College for the facilities provided and to Professor Sheila Sherlock for continued support.

Requests for reprints should be addressed to S.P.P.

\section{References}

Fleming, W. H., and Norman, P. S. (1960), non-enzymatic lysis of fibrin S by bile. Proc. Soc. exp. Biol. (N.Y.), 104, 636-638.

Fletcher, A. P., Biederman, O., Moore, D., Alkjaersig, N., and Sherry, S. (1964). Abnormal plasminogen-plasmin system act vity (fibrinolysis) in patients with hepatic cirrhosis: Its cause iand consequences. J. clin. Invest., 43, 681-695.

Hata, S. (1967). A plasminogen activator found in bile (Japanese). J. Physiol. Soc. Jap., 29, 116-126.

Januszko, T. (1969), The fibrinolytic activity of blood. (Polish). Rocz. Akad. Med. Marchlewskiego, Suppl. 22.

Januszko, T., and Dubinska, L. (1965), Estimation of the activator of fibrinolysis by means of the euglobulin test. Acta. med. pol., 6, 269-276.

Kulapongs, P., and Bachmann, F. (1968). Studies on the excretion of urokinase by the rat liver. Fed. Proc., 27, 569.

Kwaan, H. C., McFadzean, A. J. S., and Cook, J. (1957), On plasma fibrinolytic activity in cryptogenetic splenomegaly. Scot. med. J., 2, 137-150.

Oshiba, S., and Schoenfield, L. J. (1970). Plasminogen activator in bile stimulated by sodium taurocholate in isolated hamster livers. Proc. Soc. exp. Biol. N.Y., 133, 89-92.

Parbhoo, S. P., Kennedy, J., James, I. M., Chalstrey, L. J., Ajdukiewicz, A., Brock, P. J., Xanalatos, C., Sayer, P., and Sherlock, S. (1971). Extracorporeal pig-liver perfusion in the treatment of hepatic coma due to fulminant hepatitis. Lancet, 1, 659-665. 